\title{
Positioning Techniques in Indoor Environments Based on Stochastic Modeling of UWB Round Trip Time Measurements
}

\author{
Guido De Angelis, Senior Member, IEEE, Antonio Moschitta, Member, IEEE, and Paolo Carbone, \\ Fellow Member, IEEE
}

\begin{abstract}
In this paper, a technique for modeling propagation of Ultra Wide Band (UWB) signals in indoor or outdoor environments is proposed, supporting the design of a positioning systems based on Round Trip Time (RTT) measurements and on a particle filter. By assuming that nonlinear pulses are transmitted in an Additive White Gaussian Noise Channel, and detected using a threshold based receiver, it is shown that $\mathrm{RTT}$ measurements may be affected by a non-Gaussian noise. RTT noise properties are analyzed, and the effects of non-Gaussian noise on the performance of a RTT based positioning system are investigated. To this aim, a classical Least Square, an extended Kalman Filter and a Particle Filter are compared when used to detect a slowly moving target in presence of the modeled noise. It is shown that, in a realistic indoor environment, the Particle Filter solution may be a competitive solution, at a price of increased computational complexity. Experimental verifications validate the presented approach.
\end{abstract}

Index Terms - Ultra Wide Band; indoor positioning; TOA; tracking; Least Square; Kalman filter, Particle filter.

\section{INTRODUCTION}

Positioning techniques are a strong enabler for several application fields that include Intelligent Transportation Systems (ITSs) [1][2]. In particular, techniques to overcome some limitations of Global Navigation Satellite Systems (GNSS) were recently proposed. For instance, it was shown in [1]-[5] that, with a proper system, a vehicle may be tracked when moving in an urban canyon scenario. The approach proposed in [6] may be suitable for tracking of all those transport systems in absence of GPS signals, such as those moving in underground subways or indoor environments (eg. inside an airports or large train stations). Various communications systems have been proposed as basis for the implementation of indoor positioning systems, including Wireless Local Area Networks, (WLAN), Bluethooth, Digital Enhanced Cordless Telecommunication (DECT), Zigbee [7]-[8], cooperative localization [9]-[11] and Ultra Wide Band (UWB) [12]-[13].

In this paper, a positioning algorithm is presented, that relies on round-trip time (RTT) measurements between a mobile device and a set of fixed nodes, fed to a tracking algorithm. RTT measurements were obtained using an Ultra Wide Bandwidth (UWB) pulse communications system, that

Manuscript received Month XX, Year; revised Month Xx, 2013; accepted Month XX, 2013. Date of publication MONTH DD, YEAR; date of current version MONTH DD, YEAR. The Associate Editor for this paper was X. XXXXXXXXX.

The Authors are with the Department of Engineering, University of Perugia, via G. Duranti 93, 06125 Perugia - Italy, Email: \{antonio.moschitta,paolo.carbone\}@unipg.it, guidodeangelis@ieee.org

Digital Object Identifier XX.XXXX/TITS.XXXX.XXXXXXX extends a setup presented in [14], as described in section IIA. Different distributions and probability density functions (PDFs) have been proposed in the literature to model RTT measures obtained in a Non Line of Sight (NLoS) scenario [15]. In particular, Asymmetric Gaussian Noise is discussed in [16], while in [17] a non-Gaussian noise distribution is proposed, keeping into account various phenomena that include shadowing, NLoS communications and detector's noise [14][18][19]. In [11],[20],[21] a log-normal distribution is proposed. A mixture between a Gaussian and Uniform distribution is described in [22], while in [23] noise is modeled by adding an exponentially distributed random variable to a Gaussian one. Since the observed distribution of the measured data it is not easily predictable, the usage of a versatile PDF model is appropriate, in order to describe a wide set of experimental scenarios. The novel approach taken in this paper makes use of a Pearson distribution model to describe RTT measurement results. These are obtained using UWB pulses, and threshold based detection. The Pearson distribution is a very flexible and versatile model, includes the Gaussian distribution as a particular case, and is a useful tool when describing a non-Gaussian scenario.

Consequently, a novel measurement procedure, similar to that of [6], was proposed and applied to an indoor environment, in a planar scenario. As shown in section II-B, a preliminary calibration was carried out on a pre-existing RTT dataset, collected in a test environment and presented in [12]. Using such data, obtained by measuring RTT in a set of known positions, the stochastic properties of RTT measurement results were analyzed. In particular, a type IV Pearson distribution was identified by means of numerical fitting and used to model the measurement results [24]-[29]. Then, interpolation techniques were used to estimate the RTT noise properties in the whole indoor environment, as a function of distance between each node and the mobile node. Using such results and Montecarlo techniques, RTT measurements were simulated, feeding the results to three positioning solutions, namely a Least Square (LS) algorithm, an Extended Kalman Filter (EKF), and a Particle Filter (PF), as described in section III. Finally, results obtained with the overall Montecarlo simulation environment, including both RTT data generation and the positioning algorithms, were validated by comparing simulations to experimental data, obtained by performing RTT measurements in some additional points of the modeled environment, as discussed in section IV.

By using experimental data, the results described in this paper prove the effectiveness of the modeling strategy and show that the Particle Filter approach may be a competitive 


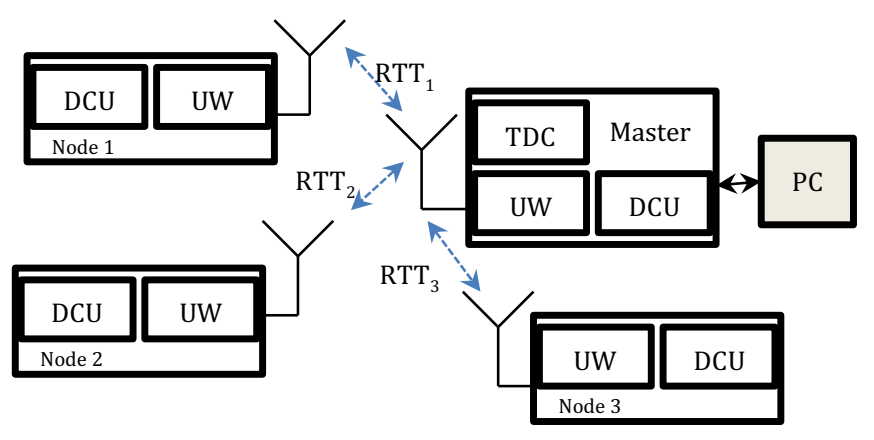

Fig. 1 - Architecture of the UWB positioning system.

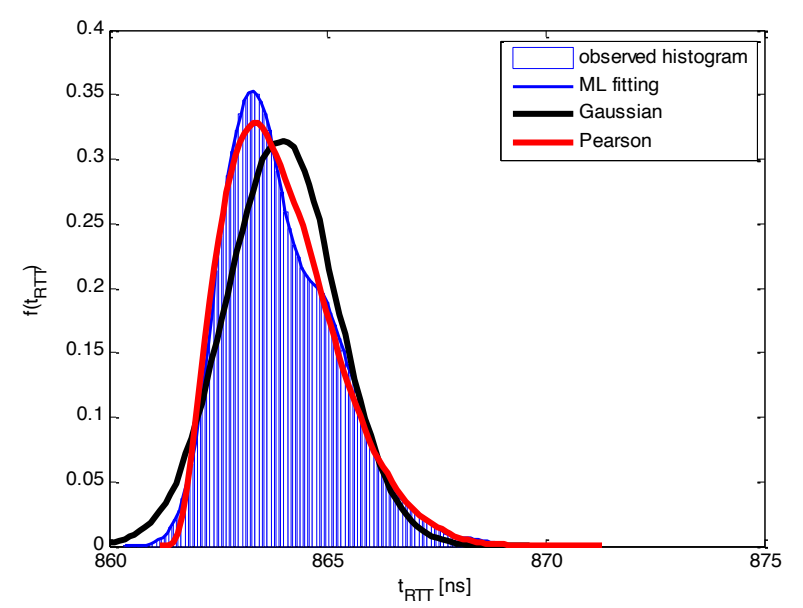

Fig. 3a - Experimental results: PDF of RTT measurements obtained during the calibration phase, for a distance $30 \mathrm{~cm}$ between the master node and a slave.

solution in indoor environments, especially when multipath effects and nonlinearities result into non Gaussian measurement noise, or when noise properties change when the mobile node moves.

\section{System Description AND RTT MEASUREMENT Model}

\section{A. System architecture}

The position measurement architecture described in this paper is based on a network of transceivers, arranged in a star topology. Line of Sight (LoS) transmission are assumed. The master device is located by measuring the RTT from a set of slave nodes, placed in known positions, as shown in Fig. 1. To this aim, it can start the RTT measurement by transmitting an UWB pulse. The slave echoes back any detected UWB pulse. Both master and slave nodes are equipped with a Digital Control Unit (DCU), used for configuration and addressing purposes. The master node measures time intervals using a Time to Digital Converter (TDC).

While UWB systems are usually produced in ASIC technology [25] [26], the adopted system is a self-produced solution, assembled using low cost COTS (Commercial Offthe-Shelf) components based on CMOS technology. Master and slave nodes use identical transceivers. The transceivers, triggered by the DCU, work in the regulated UWB frequency band between 4 and $8 \mathrm{GHz}$, and feature a homodyne architecture. Each transceiver is equipped with a circular disc antenna, printed on a dielectric substrate on the same side of the ground plane, designed to operate at a center frequency of 5.6-GHz [27]. Further design details are given in [9].

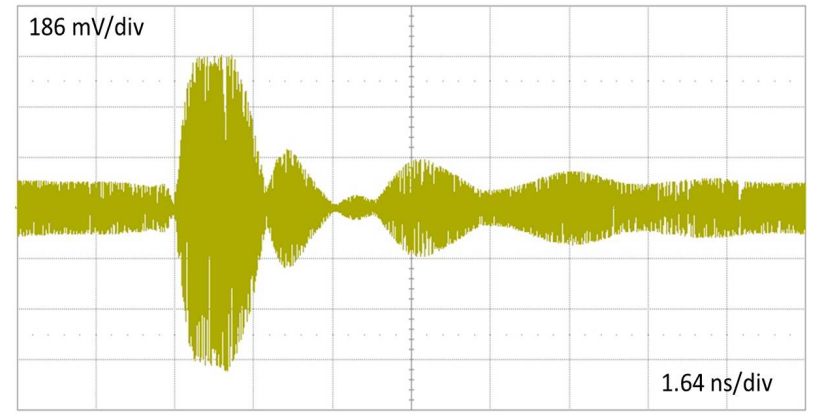

Fig. 2 - Shape of the pulse-modulated burst

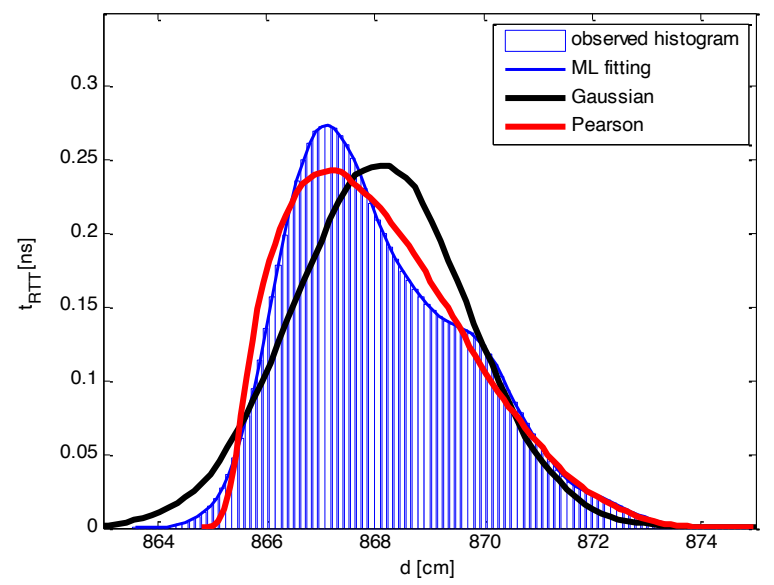

Fig. $3 b$ - Experimental results: PDF of RTT measurements obtained during the calibration phase, for a distance $70 \mathrm{~cm}$ between the master node and a slave.

Both the master and the slave nodes are equipped with an UWB receiver circuitry. The receiver mixes down the incoming signal, and identifies the recovered baseband pulse using a threshold detector [12]. The master node initiates the measurement process by enabling a slave device, subsequently transmitting an UWB pulse [8]. After being enabled, each of the three slave nodes echoes back an UWB pulse when an incoming UWB pulse is detected. Thus, by sequentially enabling one slave at a time, the master node collects three RTT measurements in a cycle [8]. Due to the RTT approach, synchronization between nodes is not needed, requiring however a preliminary calibration, in order to assess and compensate any latency introduced by each responding device. By using calibration data, RTT measurements are converted into distance estimates and transmitted to a Personal Computer (PC) that runs a positioning algorithm to provide planar localization.

\section{B. UWB pulse description and RTT measurement model}

In a pulse based UWB system the choice of the baseband pulse shape is very important [30][31], because it may significantly affect measurement accuracy. In fact, the literature shows that the pulse shape, together with the effect of the threshold detector, may significantly affect any related time interval measurement [14][32]. The UWB signal used throughout this paper is shown in Fig. 2. It is obtained by feeding a baseband pulse to a mixer that combines it with a $5.6 \mathrm{GHz}$ sinewave [11].

To obtain RTT data, the system developed in [12] was preliminarily calibrated in semi-anechoic chamber, in order 


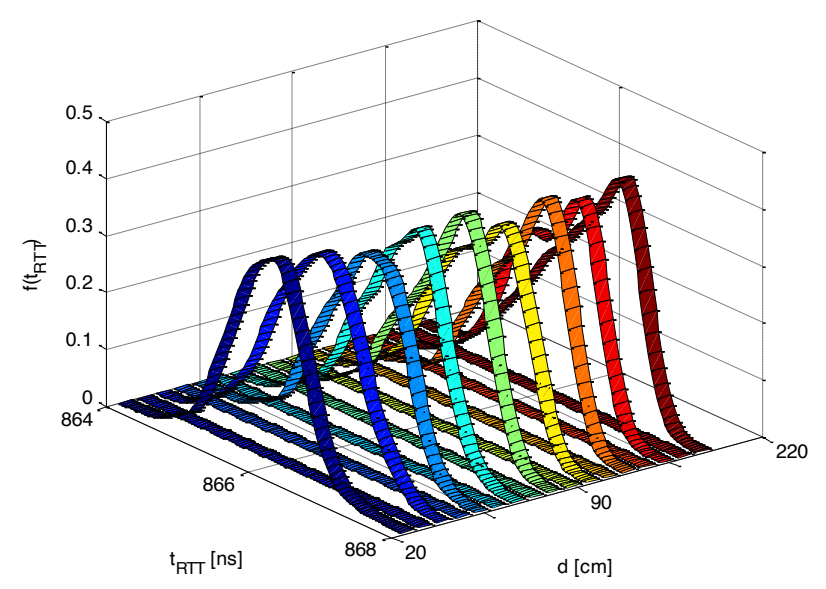

Fig. 4 - Observed distribution of $t_{R T T}$, at different distances

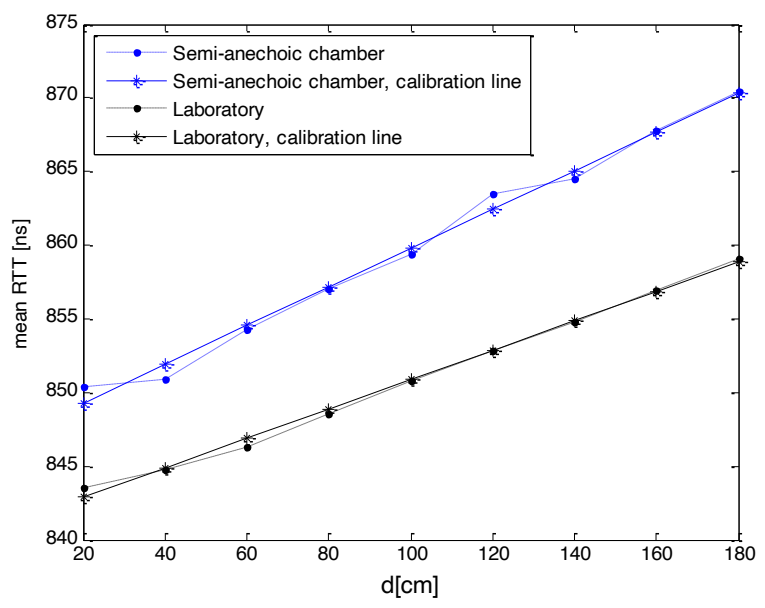

Fig. 5 - Mean value or $t_{R T T}$ and calibration lines, obtained both in a laboratory (black) and in a semi-anechoic chamber (blue).

to model the distribution of the collected measures under LOS conditions, and in absence of multipath.

In particular, $N=5 \cdot 10^{3}$ RTT measurement results were collected by placing the master and each slave at known distances, ranging from $20 \mathrm{~cm}$ to $180 \mathrm{~cm}$, and by estimating, for each distance, the PDF. The observed RTT distributions were fitted to a type IV Pearson distribution, given by [33][34]:

$$
f(x)=k\left(1+\left(\frac{x-\lambda}{a}\right)^{2}\right)^{-m} e^{-v \cdot \operatorname{arctg}\left(\frac{x-\lambda}{a}\right)},
$$

where $k$ is a normalizing constant, given by

$$
k=\left|\frac{\Gamma\left(m+\frac{v}{2} i\right)}{\Gamma(m)}\right|^{2}\left(\alpha B\left(m-\frac{1}{2}, \frac{1}{2}\right)\right)^{-1},
$$

$\Gamma(\cdot)$ is the complex Gamma function, and $B(\cdot)$ is the Beta function [24]. By assuming $r=2(m-1)$, the parameters $a, m, \lambda$ and $v$ can be related to the mean value $\mu$, the variance $\sigma^{2}$, the skewness $\gamma_{1}$ and the kurtosis $\gamma_{2}$ by the following [34]

$$
\mu=\lambda-\frac{a v}{2(m-1)},
$$
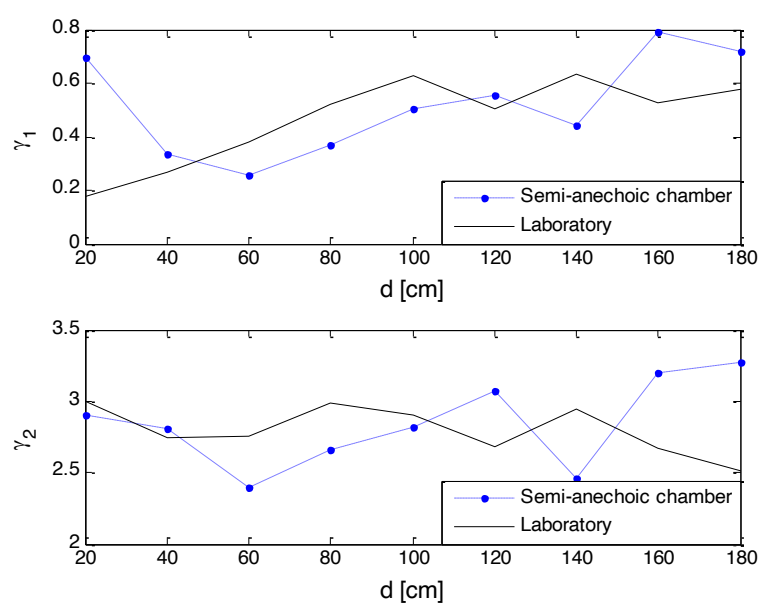

Fig. 6 - Skewness $\gamma_{1}$ and kurtosis $\gamma_{2}$, as a function of the masterslave distance $d$.

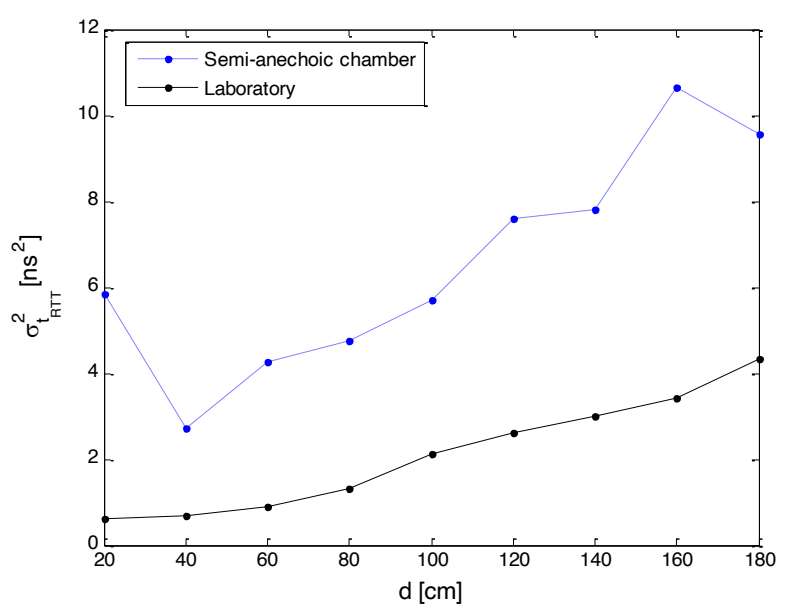

Fig. 7 - Measured RTT variance, as a function of the master-slave distance $d$.

$$
\begin{aligned}
& \sigma^{2}=\frac{a^{2}\left(r^{2}+v^{2}\right)}{r^{2}(r-1)}, \\
& \gamma_{1}=\frac{-4 v}{r-2} \sqrt{\frac{r-1}{r^{2}+v^{2}}}, \\
& \gamma_{2}=\frac{3(r-1)\left[(r+6)\left(r^{2}+v^{2}\right)-8 r^{2}\right]}{(r-2)(r-3)\left(r^{2}+v^{2}\right)},
\end{aligned}
$$

The fitting was completed by evaluating the sample mean, variance, skewness and kurtosis from the $N$ RTT positions, inverting (3)-(6) to obtain $\mathrm{r}, a, m, \lambda$ and $v$, given by

$$
\begin{aligned}
& r=\frac{6\left(\gamma_{2}-\gamma_{1}^{2}-1\right)}{2 \gamma_{2}-3 \gamma_{1}^{2}-6}, \\
& v=-\frac{r(r-2) \gamma_{1}}{\sqrt{16(r-1)-\gamma_{1}^{2}(r-2)^{2}}}, \\
& a=\frac{\sqrt{\sigma^{2}\left(16(r-1)-\gamma_{1}^{2}(r-2)^{2}\right)}}{4}, \\
& \lambda=\mu-\frac{(r-2) \sigma \gamma_{1}}{4} .
\end{aligned}
$$


For instance, Fig. 3a shows a histogram, describing the RTT distributions obtained for a master-slave distance of 30 $\mathrm{cm}$. Three curves are also shown. The first one (blue) is the envelope of the observed histogram, obtained using a maximum likelihood fitting. The second one (black) is obtained by fitting the data to a Gaussian distribution. The third one (red) is obtained by fitting the data to a type IV Pearson distribution respectively. Similar results were obtained for a distance $d$ of $70 \mathrm{~cm}$, as shown in Fig. 3b. Figs $3 \mathrm{a}$ and $3 \mathrm{~b}$ suggest that the type IV Pearson distribution is a good candidate for PDF modeling, since it can easily describe both Gaussian and non-Gaussian asymmetric distributions, depending on the values assumed by its various parameters. Fig. 4 shows RTT distributions measured for various distances. It can be observed that the asymmetry of the observed distribution increases with $d$. In particular, the four parameters $\mu, \sigma^{2}, \gamma_{1}$ and $\gamma_{2}$ were estimated using fitting techniques, for each considered distance, collecting measurements both in a laboratory and in a semi-anechoic chamber. The RTT mean value $\mu$ was measured and plotted in Fig. 5 as a function of distance between the master and the slave nodes. As expected, the mean RTT linearly grows with the distance $d$, the slope and the intercept keeping into account both propagation and detection delays [14][35]. Fitting lines were determined, and shown as well. Moreover, Fig. 6 shows the skewness $\gamma_{1}$ and the kurtosis $\gamma_{2}$, for RTT measurements taken between the master and one of the slave nodes at different distances. The mean value of kurtosis observed at various distances in the semi-anechoic chamber is about 2.84 , while the skewness is positive, with a mean value of 0.52 . These values significantly differ from those of Gaussian distribution, and motivate the usage of a type IV Pearson distribution, to conveniently describe the observed data. To further characterize the RTT distribution, RTT variance was measured as well, both in a laboratory and in a semianechoic chamber as a function of distance between master and slave nodes. As expected, the RTT variance grows with distance, because of the reduced signal to noise ratio at the receiver input [29]. The behavior of the measured RTT variance $\sigma_{t_{R T T}}^{2}$ has been approximated with the simple linear law.

$$
\sigma_{t_{R T T}}^{2} \cong \alpha d+\beta,
$$

where, for RTT measurements taken in a laboratory, $\alpha=0.024 \mathrm{~ns}^{2} / \mathrm{cm}$ and $\beta=-0.2788 \mathrm{~ns}^{2}$ were obtained using numeric fitting in the considered distance range. A greater variance was observed in the semi-anechoic chamber than in the laboratory, as shown in Fig. 7. Such difference was investigated, and ascribed to a higher operating temperature of the UWB System, with respect to the laboratory experiments, as described in [36]. Finally, in order to further assess the usefulness of the Pearson Type IV distribution in modeling RTT measurements, the Kullback-Leibler Divergence (KLD) between the observed distribution, represented by the normalized histograms, and the considered models, fitted to the measured values of $\mu, \sigma_{t_{R T T}}^{2}$, $\gamma_{1}$ and $\gamma_{2}$, for various distances between the master and the slave nodes. Fig. 8 shows the KLD obtained from measurement results collected in the semi-anechoic chamber (a), and in the laboratory (b). For measurements collected in the semi-anechoic chambers the Pearson KLD is below 0.02 in all considered cases, and for measurements taken in the laboratory is below the same threshold in all measurement points but one. It can also be observed that the Pearson Type IV model systematically leads to a lower KLD than the Gaussian one, and that the difference between the two KLDs grows with distance. This result suggests that replacing the Gaussian noise model with the Pearson Type IV model may lead to more realistic simulation results. This issue is further discussed in Section III-A.

In the following activities, the laboratory calibration data have been used to describe RTT variance, because they describe a more realistic operating scenario.

\section{Distance Measurement Model}

Time of Flight measurements on pulsed signals are often robust to multipath, because the Line of Sight (LoS) contribution can be discriminated. In fact, the response of the multipath environment can be modeled by a train of signal replicas, the first of which is usually the LoS one. Even if the LoS pulse and its replicas overlap in time, they can often be discriminated using correlation techniques. Thus, if LoS path is available and in absence of significant multipath effects, the RTT can be modeled as [14]:

$$
t_{R T T}=k_{R T T} d+t_{0}+n_{R T T},
$$

where $d$ is the distance between the master and slave nodes, $t_{0}$ is a fixed latency contribution, $k_{R T T}$ is a slope factor that keeps into account both propagation and variable detection delays, and $n_{R T T}$ is measurement noise, modeled in the previous section using the type IV Pearson distribution. Notice that $k_{R T T}$ and $t_{0}$ can be approximated by the slope and the intercept of the fitting line describing laboratory calibration in Fig. 4. In particular, the results of laboratory calibration correspond to $k_{R T T} \cong 0.1 \mathrm{~ns} / \mathrm{cm}$ and $t_{0} \cong 840.87 \mathrm{~ns}$. Equation (12) can be inverted, leading to [8]

$d=\frac{t_{R T T}-t_{0}}{k_{R T T}}+n_{0}, \quad n_{0}=\frac{n_{R T T}}{k_{R T T}}$,

where $n_{0}$ is a scaled version of $n_{R T T}$, thus featuring a similar distribution. By neglecting the noise contribution, the distance can be estimated as

$$
\hat{d}=\frac{\hat{t}_{R T T}-t_{0}}{k_{R T T}} \text {. }
$$

Moreover, since (14) is an affine transformations, if the RTT is described by a Type IV Pearson distribution, the estimated distance $d$ can be described by the same distribution, with a mean value shifted by $-t_{0} / k_{R T T}$ and variance given by

$$
\sigma_{\hat{d}}^{2} \cong \frac{1}{k_{R T T}^{2}} \sigma_{t_{R T T}}^{2} \cong \frac{1}{k_{R T T}^{2}}(\alpha d+\beta) .
$$

Notice that, since the noise mean value and variance depend on the position, when the mobile node is moving, the distribution of the measurement results is non-stationary. Practical implications of this statement are discussed in section IV.

\section{CONSIDERED POSITIONING ALGORITHMS}

In order to identify the most effective positioning strategy, some algorithms have been compared, assuming a slowly moving mobile node, such that the time required to 

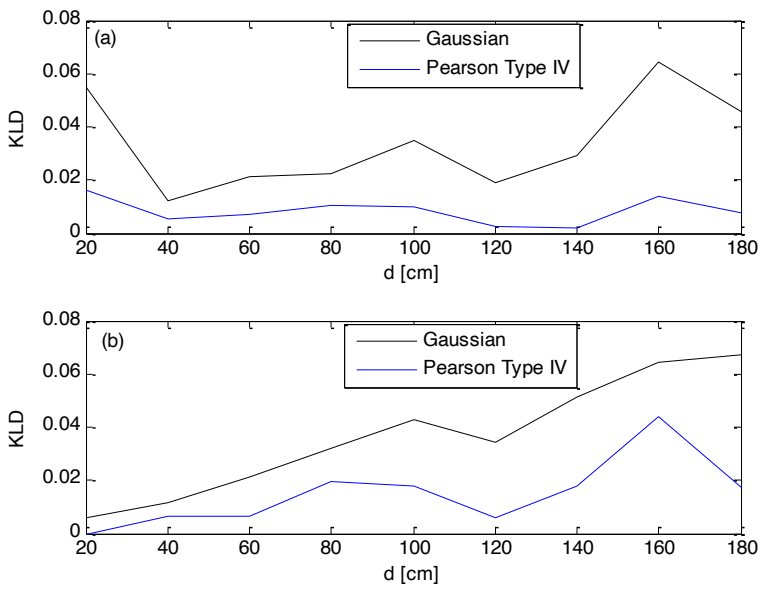

Fig. 8 - Experimental results: Kullback-Leibler divergence between the observed distribution and the considered models as a function of the master-slave distance $d$, obtained for data collected in the semianechoic chamber (a), and in a laboratory (b).

collect distance measurements and run the triangulation algorithm corresponds to a negligible displacement of the mobile node. We also assumed that the repeated distance measurements were available for each position taken by the mobile node. Under such conditions, three algorithms were compared, the Least Squares, the Extended Kalman Filter, and the Particle Filter, described in the following subsections.

\section{A. Least Square (LS)}

Let us define $\hat{d}_{R T T, i}$ as the RTT based estimation of the Euclidean distance $d_{u, i}$ between the mobile node, located in $\left(x_{u}, y_{u}\right)$, and the $i$-th anchor, located in $\left(x_{i}, y_{i}\right)$, given by

$$
d_{u, i}=\sqrt{\left(x_{u}-x_{i}\right)^{2}+\left(y_{u}-y_{i}\right)^{2}}, \quad i=1, \ldots, N .
$$

Approximating (12) with a $1^{\text {st }}$ order Taylor expansion about a reference point $\left(x_{v}, y_{v}\right)$ results into

$$
d_{u, i}=d_{v, i}+a_{i, 1} \delta_{x}+a_{i, i} \delta_{y}, \quad i=1, \ldots, N,
$$

where $\delta x=x_{u}-x_{v}, \delta y=y_{u}-y_{v}, N$ is the number of anchors, and $d_{v, i}$ is the distance between the reference point and the $i$-th anchor, given by

$$
d_{v, i}=\sqrt{\left(x_{v}-x_{i}\right)^{2}+\left(y_{v}-y_{i}\right)^{2}}, \quad i=1, \ldots, N,
$$

while the sensitivities $a_{i, 1}$ and $a_{i, 2}$ are respectively given by

$a_{i, 1}=\left.\frac{\partial d_{u, i}}{\partial x_{u}}\right|_{\left(x_{u}, y_{u}\right)=\left(x_{v}, y_{v}\right)}=\frac{x_{v}-x_{i}}{d_{v, i}}$,

and

$$
a_{i, 2}=\left.\frac{\partial d_{u, i}}{\partial y_{u}}\right|_{\left(x_{u}, y_{u}\right)=\left(x_{v}, y_{v}\right)}=\frac{y_{v}-y_{i}}{d_{v, i}} .
$$

By approximating $d_{u, i}$ in (17) with its RTT based estimation $\hat{d}_{R T T, i}$, we obtain a system of $N$ equations

$a_{i, 1} \delta_{x}+a_{i, 2} \delta_{y}=\hat{d}_{R T T, i}-d_{v, i}, \quad i=1, \ldots, N$,
$A \delta=D$,

where

$$
A=\left|\begin{array}{cc}
a_{1,1} & a_{1,2} \\
a_{2,1} & a_{2,2} \\
\vdots & \vdots \\
a_{N, 1} & a_{N, 2}
\end{array}\right|, D=\left|\begin{array}{c}
\hat{d}_{R T T, 1}-d_{v, 1} \\
\hat{d}_{R T T, 2}-d_{v, 2} \\
\vdots \\
\hat{d}_{R T T, N}-d_{v, N}
\end{array}\right|, \delta=\left[\delta_{x}, \delta_{y}\right]^{T} .
$$

Solving (18) using the pseudo-inverse method leads to the LS solution, estimating $\delta$ and then the mobile position as

$\hat{x}_{u}=v_{v}+\hat{\delta}_{x}, \hat{y}_{u}=y_{v}+\hat{\delta}_{y}$.

The estimation was implemented using an iterative and recursive procedure, by selecting a point in the middle of the measurement environment as reference point of the first iteration, and, for the following iterations, the solution obtained at the (n-1)-th iteration as reference point for the $n$ th iteration. Repeated empirical tests suggested that 60 cycles are enough for the algorithm to converge.

\section{B. Extended Kalman Filter}

Given a suitable sampling time $T_{C}$ and an integer sample index $k$, the mobile node motion can be described, at time $t_{k}=k T_{C}$, by its position and velocity, that is by the state vector sequence

$x_{k}=\left[x_{u, k}, y_{u, k}, \dot{x}_{u, k}, \dot{y}_{u, k}\right]^{T}$,

where $x_{u, k}$ and $y_{u, k}$ describe the mobile node position, while $\dot{x}_{u, k}$ and $\dot{y}_{u, k}$ are the coordinates of the corresponding velocity vector. The state vector evolution can be described as

$$
x_{k+1}=f\left(x_{k}, w_{k}\right),
$$

where $w_{k}$ is the model noise at time index $k$, with a $4 \times 4$ correlation matrix $Q$. For the considered motion problem, the model $f(\cdot, \cdot)$ can be assumed linear and the model noise can be assumed as

$x_{k+1}=F x_{k}+w_{k}$,

where $F$ is the state transition matrix and $Q$ the process noise covariance as [15], given by

$F=\left|\begin{array}{cccc}1 & 0 & T_{C} & 0 \\ 0 & 1 & 0 & T_{C} \\ 0 & 0 & 1 & 0 \\ 0 & 0 & 0 & 1\end{array}\right|, Q=q\left|\begin{array}{cccc}\frac{T_{C}^{3}}{3} & 0 & \frac{T_{C}^{2}}{2} & 0 \\ 0 & \frac{T_{C}^{3}}{3} & 0 & \frac{T_{C}^{2}}{2} \\ \frac{T_{C}^{2}}{2} & 0 & T_{C} & 0 \\ 0 & \frac{T_{C}^{2}}{2} & 0 & T_{C}\end{array}\right|$,

where $T_{C}$ is the sample period and $q$ denotes the power spectral density of the process noise.

Let us also assume that a sequence $z_{k}$ of measurements is collected, related to the state vector by the following

$$
z_{k}=h\left(x_{k}, v_{k}\right)
$$

where $v_{k}$ is the $k$-th sample of measurement noise, usually assumed additive and with a diagonal autocorrelation matrix $R$. If the measurement model $h(\cdot, \cdot)$ is linear, (29) reduces to

that may be represented in matrix form as 


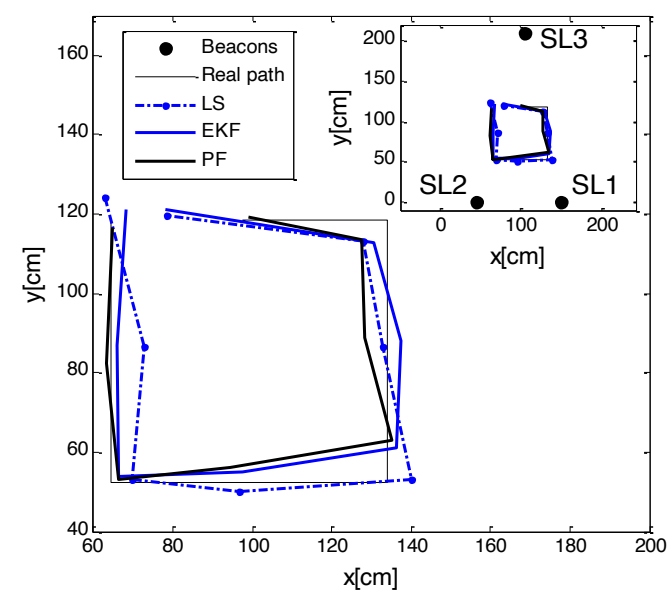

Fig. 9. Experimental results: position estimation based on various algorithms used to process RTT measurement results. SL1, SL2, and SL3, shown in the upper right inset, are the 3 slave nodes, acting as beacons. The thin black curve shows the true positions, the solid black, solid blue, and dashed blue curves respectively show the positions estimated using the PF, EKF, and LS algorithms.

$z_{k}=H x_{k}+v_{k}$,

where $H$ is the system observation matrix. Notice that, for the considered problem, the measurement vector is given by

$z_{k}=\left[\hat{d}_{R T T, 1}, \hat{d}_{R T T, 2}, \ldots, \hat{d}_{R T T, N}\right]$.

Moreover, the non-linear measurement model associated to distance measurements (16) can be linearly approximated, assuming

$H=\left|\begin{array}{cccc}\frac{\partial d_{u, 1}}{d x_{u}} & \frac{\partial d_{u, 1}}{d y_{u}} & 0 & 0 \\ \vdots & \vdots & \vdots & \vdots \\ \frac{\partial d_{u, N}}{d x_{u}} & \frac{\partial d_{u, N}}{d y_{u}} & 0 & 0\end{array}\right|=\left|\begin{array}{cccc}a_{1,1} & a_{1,2} & 0 & 0 \\ \vdots & \vdots & \vdots & \vdots \\ a_{N, 1} & a_{N, 2} & 0 & 0\end{array}\right|$

the sensitivities $a_{i, 1}$ and $a_{i, 2}$ being given by (19) and (20). The matrix $H$ is updated at each time step, using the current predicted state $\left(x_{u}, y_{u}\right)$ as an input.

The Extended Kalman Filter (EKF) computes first an $a$ priori state estimation $\hat{x}_{k}^{-}$, based on past system history, and then updates $\hat{x}_{k}^{-}$by properly weighting the innovation, represented by measurements $z_{k}$, leading to an a posteriori state estimation $\hat{x}_{k}$. By minimizing the covariance $P_{t}$ of the a posteriori error $e_{k}=x_{k}-\hat{x}_{k}$, a recursive estimation algorithm is obtained, shown in Table I, where $K_{t}$ is known as the Kalman gain at time index $k$. The EKF algorithm can be initialized by properly setting an initial value for the $a$ priori error covariance matrix $P_{k}^{-}$.

Table I - Kalman Filter algorithm

1. $\hat{x}_{k}^{-}=F \hat{x}_{k-1}$

2. $P_{k}^{-}=F P_{k-1} F^{T}+Q$

3. $K_{k}=P_{k}^{-} H^{T}\left(H P_{k}^{-} H^{T}+R\right)^{-1}$

4. $\hat{x}_{k}=\hat{x}_{k}^{-}+K_{k}\left(z_{k}-H \hat{x}_{k}^{-}\right)$

5. $P_{k}=P_{k}^{-}-K_{k} H P_{k}^{-}$

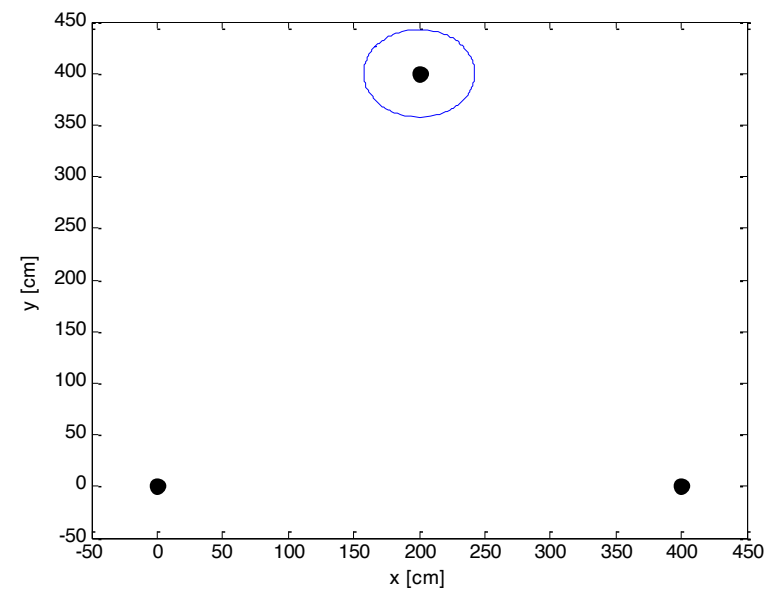

Fig. 10. A simulated position estimation scenario, assuming that the mobile node moves in a narrow circle centered on a beacon.
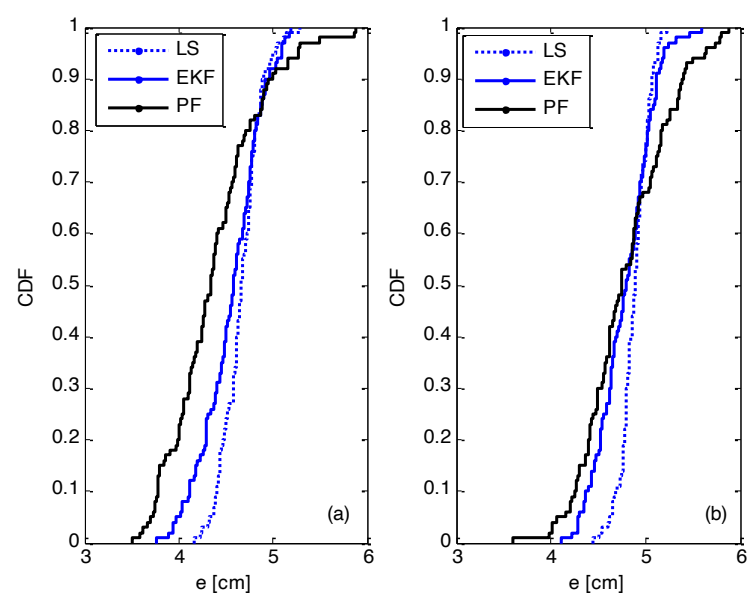

Fig. 11. CDF of the positioning error obtained for the scenario depicted in Fig. 10, using the considered positioning algorithms. The left plot (a) was obtained assuming that the RTT measurements in a fixed position are Gaussian distributed. The right plot (b) was obtained assuming the more accurate Pearson Type IV model.

\section{Particle filter}

The Particle Filter is a popular numerical method, effective for solving non-linear and non-Gaussian problems, and considered advantageous compared to the extended Kalman filter, since it is sub-optimal for non-Gaussian distributions. The price paid is a higher computational complexity. Applications of PFs to tracking problems have been discussed in [37]-[44]. In the following a tracking application is shown, based on RTT measurements collected using the UWB system [12], or simulated using the same statistical distribution. In particular, the standard motion model presented in [6] was adopted with a 4-state vector representing planar position coordinates and velocities at time $k$ as

$$
\begin{aligned}
& x_{u, k+1}=x_{u, k}+\dot{x}_{u, k} T_{C}+\omega_{x, k} \frac{T_{C}^{2}}{2}, \\
& \dot{x}_{u, k+1}=\dot{x}_{u, k}+\omega_{x, k} T_{C}, \\
& y_{u, k+1}=y_{u, k}+\dot{y}_{u, k} T_{C}+\omega_{y, k} \frac{T_{C}^{2}}{2}, \\
& \dot{y}_{u, k+1}=\dot{y}_{u, k}+\omega_{y, k} T_{C},
\end{aligned}
$$

where the process noise components $\omega_{x, k}$ and $\omega_{y, k}$ are assumed independent to each other and possibly not 
Gaussian. In matrix form we have

$\left|\begin{array}{l}x_{k+1} \\ y_{k+1} \\ \dot{x}_{k+1} \\ \dot{y}_{k+1}\end{array}\right|=\left|\begin{array}{cccc|c}1 & 0 & T_{C} & 0 \\ 0 & 1 & 0 & T_{C} \\ 0 & 0 & 1 & 0 \\ 0 & 0 & 0 & 1\end{array}\right|\left|\begin{array}{c}x_{k} \\ y_{k} \\ \dot{x}_{k} \\ \dot{y}_{k}\end{array}\right|+\left|\begin{array}{l}\omega_{x, k} T_{C} \\ \omega_{y, k} T_{C} \\ \omega_{x, k} \frac{T_{C}^{2}}{2} \\ \omega_{y, k} \frac{T_{C}^{2}}{2}\end{array}\right|$.

Particle filters operate by estimating the a-posteriori distribution describing the space state. The samples of such distribution are called particles, where each particle $\kappa_{k}^{m}$ (with $1 \leq m \leq M)$ is a concrete instantiation of the vector state at the time $k, M$ is the number of particles, and $\chi_{k}^{m}=\left[\kappa_{k}^{1}, \kappa_{k}^{2}, \ldots, \kappa_{k}^{M}\right]$ is an instantiation of the particle set $c_{k}$, describing the a-posteriori distribution. At each new time, particles are updated and weighted by a set of coefficients $w_{k}^{m}$.

The PF algorithm was implemented as in [6] and [39], assuming $M=2500$. The particles were initialized by uniformly sampling a disk region, including all the slave nodes, leading to procedure of Table II.

Table II - Particle Filter Algorithm [6]

(PF) The particles are initialized sampling uniformly in a disk region comprising all beacons. At each step $k \in\{1,2, \ldots, T\}$ do:

1. Apply the motion model defined above to each particle, which gives $\chi_{k+1}^{m}$ as a function of $\chi_{k}^{m}$ and the current samples $\omega_{x, k}^{m}, \omega_{y, k}^{m}$.

2. Apply the measurement model to each particle and normalize the weights.

3. Resample and estimate the position as the mean of the new (resampled) set of particles.

\section{III.EXPERIMENTAL AND SIMULATION RESULTS}

The positioning problem was analyzed in a planar context, assuming to operate in LoS conditions, characterizing and comparing to each other the considered positioning and tracking techniques. To carry out a fair comparison, all algorithms were fed with the same distance estimations, between a mobile node and a set of 3 anchors, placed in known position. In a first phase, described in subsection III.A, the PF accuracy was evaluated using a set of RTT measurements between a mobile node and a set of known position anchors, collected in a laboratory environment and used to provide distance estimations using the setup described in [12]. In a second phase, described in subsection III.B, the PF was compared against LS and EKF using a larger dataset, obtained from a simulation model. The model was developed by observing that, since according to (12)(15) the distance and the RTT measurements are linearly related, their PDF can both be described by a Pearson Type IV distribution. Thus, for each point of the $x-y$ plane of a simulated environment, the distance measurement was modeled as a realization of a Type IV Pearson random variable. For each considered point, the mean value of the Pearson distribution was assumed equal to the Euclidean distance $d_{u, i}$ between the mobile node, located in $\left(x_{u}, y_{u}\right)$, and each beacon, located in $\left(x_{i}, y_{i}\right)$, given by (16), while skewness, kurtosis and variance were obtained from those of the RTT distribution, estimated from calibration activities described in section II. In the following, the position of the mobile node was assumed to change every second, and accordingly a measurement rate of $1 \mathrm{~Hz}$ was assumed.

\section{A.Experimental results}

The PF algorithm was initially used to infer the master node position from a dataset of distance estimates, created by progressively relocating the master node in a set of know positions in a laboratory environment, and obtaining for each positions 100 distance estimations from RTT measurements. Such dataset was chosen for comparison purposes, since it had been obtained in a previous activity described in [12], where a LS algorithm was used for positioning purposes. Due to the small number of positions and to the portable context, the model (32) and (33) was simplified, implementing the Particle Filter for a reduced state $\chi=[x, y]$. An extended Kalman Filter was also considered, again based on the same reduced space state adopted for the PF. Moreover, for equity of comparison between the 3 algorithms, outliers in the range dataset were not identified and discarded prior applying the position estimation as in [12].

In order to evaluate the PF accuracy the estimated positions were plotted in Fig. 9 for each algorithm, together with the true ones (black curve). With respect to Fig. 9, where the coordinates are expressed in $\mathrm{cm}$, the three slaves were positioned in $(181,0),(45,0)$ and $(105,210)$ respectively. Three dashed curves are also shown, describing the positions estimated using the PF (black), the EKF (solid blue), and the LS (dashed blue) algorithms. The positions of the three beacons are shown in the zoomed out upper right inset. For each algorithm, the positioning error, defined as the Euclidean distance between the estimated position and the true one was very low for most positions, while accuracy worsened in the rightmost positions, the last to be estimated. Such a behavior was subsequently ascribed to progressive heating of the UWB system and possibly to small geometrical asymmetries of the layout, also because the adopted UWB antennas are anisotropic [12].

The simplified PF provided an error mean value of 5.14 $\mathrm{cm}$, a slightly better performance than that obtained using the same dataset and the LS algorithm, leading to an error mean value of $7.02 \mathrm{~cm}$, and the EKF, corresponding to an error mean value of $8.15 \mathrm{~cm}$. Since in the considered linearized problem the LS and the Kalman filter are expected to provide nearly optimal performance in presence of Gaussian noise, this result also suggests that the Pearson Type IV model can better describe measurement results than the Gaussian one.

\section{B.Simulation results}

The result in Fig. 4 and Fig. 8 suggest that both the RTT measurement results and the range estimations tend to be non-Gaussian when the distance between the mobile node and the beacons becomes large. On the other hand, data in Fig. 9 suggest that the PF can be competitive with respect to LS and EKF estimators when measurements are not Gaussian distributed. Observe that, even if the range measurements between the mobile node and a given beacon could be suitably described by a Gaussian distribution, this 


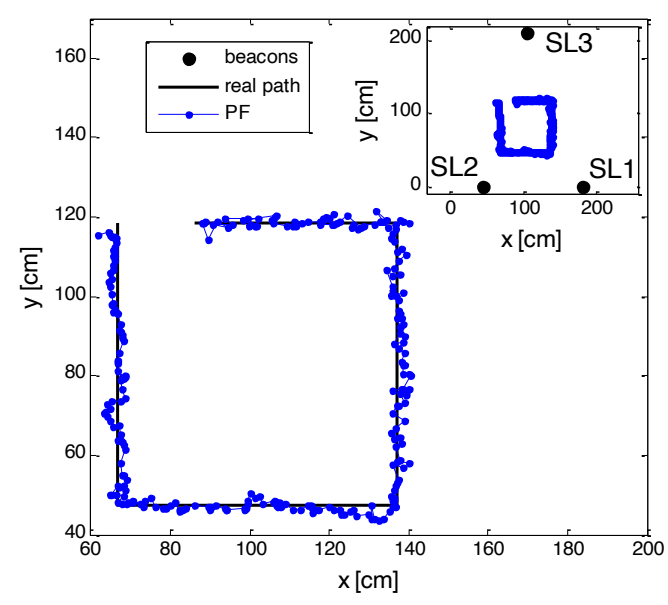

Fig. 12. Position estimation, carried out using the PF to process RTT data, obtained for a simulated environment. SL1, SL2, and SL3 are the 3 slave nodes, acting as beacons. The black curve shows the true positions, the blue curve shows the positions estimated using the PF.

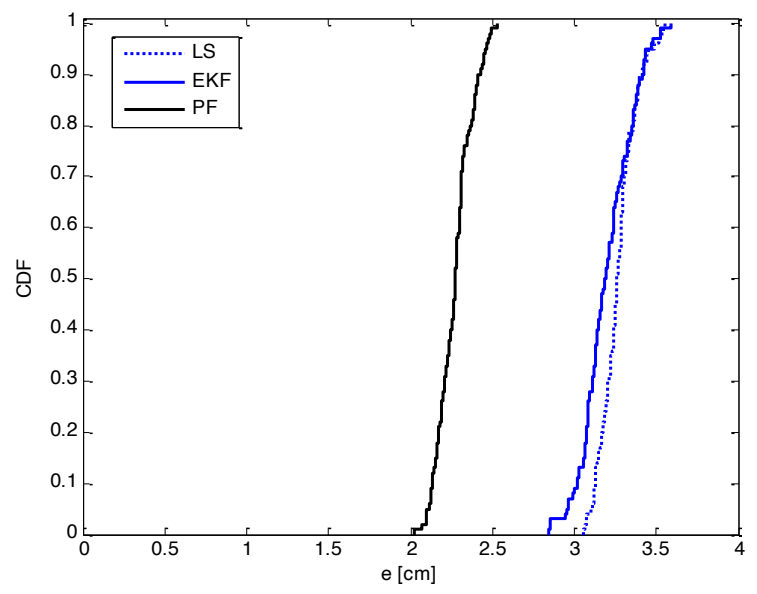

Fig. 13. CDF of the positioning error obtained for the scenario depicted in Fig. 12, using the considered positioning algorithms.

would not ensure an overall Gaussian distribution when the mobile node position moves on a trajectory. In fact, as observed in section II, in this case the distribution of the measurement noise is no longer stationary. Thus, combining Gaussian results with different mean values and variances can easily lead to non-Gaussian distributions. To further investigate this issue, a position measurement was simulated, assuming that the mobile node moves in a narrow circle about a slave, as shown in Fig. 10. In this case, the distribution of the range measurement noise can be considered almost stationary, because the considered positions are very close to each other.

Thus, for each position assumed by the mobile node, distance measurements were simulated, first by assuming a Gaussian distribution, and then by assuming a Pearson Type IV distribution. For each considered distance between the mobile node and the beacons, the mean value $\mu_{\hat{d}}$, and the standard deviation $\sigma_{\hat{d}}^{2}$ of the range estimations were selected using (12)-(15). The Pearson Type IV model was completed by assuming a skewness $\gamma_{1}=0.52$ and a kurtosis $\gamma_{2}=2.84$, deduced from the results in Fig. 6. Under such conditions, the cumulative distribution function (CDF) of the positioning error $e$ was evaluated, for the LS, EKF, and PF estimators respectively, leading to the results in Fig. 11a, obtained by modeling range measurements in a fixed position as Gaussian distributed, and $11 \mathrm{~b}$, obtained by assuming a Pearson Type IV distribution. It can be observed that the PF provides comparable performance with respect to the EKF and the LS, even if the range measurements are Gaussian distributed. The measurement of a fixed position was considered as well, showing that, when range measurements are Gaussian distributed, the LS and the EKF provide much more accurate results than the PF. Consequently, the Gaussian model may be inadequate for the problem at hand, especially in a motion tracking scenario.

Following these results, a larger data set of 268 positions was developed using a simulation model, in which distance measurements were simulated for a set of points with respect to the same three known position beacons considered in Fig. 9. Calibration results were used to set the parameters of three Type IV Pearson distributions, each used to model the distance estimation between the mobile node and each of the beacons. In particular, we selected again a skewness $\gamma_{1}=0.52$, a kurtosis $\gamma_{2}=2.84$, a mean value equal to the true Euclidean distance $d$ between the assumed position and a beacon, and we set the variance as a linear function of $d$, given by (15). Such distance estimations were then used to feed the considered positioning algorithms. This time, a full space state description was used, assuming $\chi=[x, y, \dot{x}, \dot{y}]$. Under the mentioned conditions the PF provided a good performance, shown in Fig. 12, where again both the ideal mobile node trajectory (black) and the estimated trajectory (blue) are shown.

Finally, in order to compare the various algorithms, the CDF of the positioning error $e$, was estimated for the PF, EKF, and LS algorithms across the set of considered positions assumed by the mobile node, leading to the results in Fig. 13. Similarly to Fig. 11, Fig. 13 shows that, when the RTT and the range measures are not Gaussian distributed, EKF may not be a competitive solution, because, despite its increased computational burden, the performance improvement is very similar to that of the LS approach. The $\mathrm{PF}$ provides a lower estimation error, also more independent of the position of the master-slave node. Therefore, the PF strategy may be a good candidate when the measurement model is not Gaussian. This may be the case in motion tracking problems, when the beacons' position is not regular or accurately known, or because of multipath and pulse shape [22].

\section{CONCLUSIONS}

A simple and cost effective ranging positioning technique has been presented, based on a particle filter applied to distance measurements, fed by distance estimations, obtained using RTT measurements from an UWB system and calibration results. A simulation model was developed, using calibration data to describe distance measurements between a mobile node and a set of fixed beacons with a Type IV Pearson distribution. The Particle filter has been compared against LS and against an EKF using a set of experimental data, providing slightly better performance at a price of increased computational complexity. Further comparisons against the LS method and the Kalman filter, based on synthetic data obtained from the developed simulation model, show that the particle filter may be a very competitive solution in case of non-Gaussian distributed measurements, that may occur due to transmitted signal 
properties or due to multipath phenomena, or in a motion tracking scenario, such the distribution properties change with the mobile node position.

\section{REFERENCES}

[1] Fei-Yue Wang, Daniel Zeng, Liuqing Yang, "Smart Cars on Smart Roads: An IEEE Intelligent Transportation Systems Society Update," IEEE Pervasive Computing, vol. 5, no. 4, pp. 68-69, October-December, 2006.

[2] Fengzhong Qu; Fei-Yue Wang; Liuqing Yang, "Intelligent transportation spaces: vehicles, traffic, communications, and beyond," in Communications Magazine, IEEE , vol.48, no.11, pp.136-142, November 2010.

[3] Xiang Cheng; Xiaoya Hu; Liuqing Yang; Husain, I.; Inoue, K.; Krein, P.; Lefevre, R.; Yaoyu Li; Nishi, H.; Taiber, J.G.; Fei-Yue Wang; Yabing Zha; Wen Gao; Zhengxi Li, "Electrified Vehicles and the Smart Grid: The ITS Perspective," in Intelligent Transportation Systems, IEEE Transactions on, vol.15, no.4, pp.1388-1404, Aug. 2014.

[4] G. De Angelis, G. Baruffa, S. Cacopardi, "Hybrid Positioning System for Mobile Users in Urban Scenarios," IEEE Trans. on Intelligent Transportation System, 2012.

[5] Y. Zhao, "Mobile phone location determination and its impact on intelligent transportation systems," IEEE Trans. Intell. Transp. Syst., vol.1 no. 1, pp. 55-64, Mar. 2000.

[6] M. Boccadoro, G. De Angelis, P. Valigi, "TDOA Positioning in NLOS scenarios by Particle Filtering", Wireless Network, Springer, February, 2012.H.

[7] Liu, H. Darabi, P. Banerjee, J. Liu, "Survey of Wireless Indoor Positioning Techniques and Systems," IEEE Trans. Veh. on Systems, man and Cybernetics-part.C: applications and reviews., vol. 37, no. 6, pp. 1067-1080, Nov. 2007.

[8] J.-O. Nilsson, A. De Angelis, I. Skog, and P. Carbone, "Signal processing issues in indoor positioning by ultra wide band radio aided inertial navigation," in European Signal Processing Conference Proceedings, EUSIPCO, Glasgow, Scotland, Aug. 2009, pp. 216122165.

[9] M. Z. Win, A. Conti, S. Mazuelas, Y. Shen, W. M. Gifford, D. Dardari, M. Chiani, "Network localization and navigation via cooperation." Communications Magazine, IEEE, 49(5), 56-62, 2011.

[10] Z. M. Kassas, T.E. Humphreys, (2014). Observability analysis of collaborative opportunistic navigation with pseudorange measurements. Intelligent Transportation Systems, IEEE Transactions on, 15(1), 260-273.

[11] A. Conti, M. Guerra, D. Dardari, N. Decarli, \& M. Z. Win. "Network experimentation for cooperative localization," Selected Areas in Communications, IEEE Journal on, 30(2), 467-475, 2012.

[12] A. Cazzorla, G. De Angelis, A. Moschitta, M. Dionigi, F. Alimenti and P. Carbone, "A 5.6 GHz UWB Position Measurement System," IEEE Trans. on Instrumentation and Measurement, Vol. 62, No. 3, pp. 675-683, Feb. 2013.

[13] S. Maranò, W. M.Gifford , H. Wymeersch, M. Z. Win, (2010), "NLOS identification and mitigation for localization based on UWB experimental data," Selected Areas in Communications, IEEE Journal on, 28(7), 1026-1035.

[14] A. De Angelis, M. Dionigi, A. Moschitta, R. Giglietti, and P. Carbone, "Characterization and modeling of an experimental UWB pulse-based distance measurement system," IEEE Trans. Instrum. Meas., vol. 58, no. 5, pp. 1479-1486, May 2009.

[15] Y. Bar-Shalom, X. R. Li, and T. Kirubarajan, "Estimation with Applications to Tracking and Navigation.”New York, NY: John Wiley and Sons, 2001

[16] J.M. Huerta, J. M., Vidal, J., Giremus, A., \& Tourneret, J. Y. (2009). "Joint particle filter and UKF position tracking in severe non-line-ofsight situations," Selected Topics in Signal Processing, IEEE Journal of, 3(5), 874-888.

[17] Z. Shah, Malaney, R. A. (2006, May), "Particle filters and position tracking in wi-fi networks," In Vehicular Technology Conference, 2006. VTC 2006-Spring. IEEE 63rd (Vol. 2, pp. 613-617). IEEE.

[18] Alavi, B., Pahlavan, K., "Modeling of the TOA-based distance measurement error using UWB indoor radio measurements," in Communications Letters, IEEE, vol.10, no.4, pp.275-277, Apr 2006.

[19] Bartoletti, S.; Wenhan Dai; Conti, A.; Win, M.Z., "A Mathematical Model for Wideband Ranging," in Selected Topics in Signal Processing, IEEE Journal of , vol.9, no.2, pp.216-228, March 2015.
[20] M. Aso, M., Kawabata, M., \& Hattori, T. (2001). A new location estimation method based on maximum likelihood function in cellular systems. In Vehicular Technology Conference, 2001. VTC 2001 Fall. IEEE VTS 54th (Vol. 1, pp. 106-110). IEEE.

[21] I. Guvenc, C. C. Chong, F. Watanabe, "NLOS identification and mitigation for UWB localization systems," In Wireless Communications and Networking Conference, 2007. WCNC 2007. IEEE (pp. 1571-1576). IEEE, March 2007.

[22] De Angelis, A.; De Angelis, G.; Carbone, P., "Using GaussianUniform Mixture Models for Robust Time-Interval Measurement," in Instrumentation and Measurement, IEEE Transactions on, vol.PP, no.99, pp.1-1

[23] L. Cong, \& Zhuang, W. (2005)," Non line-of-sight error mitigation in mobile location," Wireless Communications, IEEE Transactions on, 4(2), 560-573.

[24] Abramowitz, M., Stegun. A., "Handbook of mathematical functions: with formulas, graphs, and mathematical tabeles," Dover Publications, 1965.

[25] Kegen Yu, Oppermann, I., "Performance of UWB position estimation based on time-of-arrival measurements," in Ultra Wideband Systems, 2004. Joint with Conference on Ultrawideband Systems and Technologies. Joint UWBST \& IWUWBS. 2004 International Workshop on, vol., no., pp.400-404, 18-21 May 2004.

[26] Stoica, L.; Rabbachin, A.; Oppermann, I., "A low-complexity noncoherent IR-UWB transceiver architecture with TOA estimation," in Microwave Theory and Techniques, IEEE Transactions on, vol.54, no.4, pp.1637-1646, June 2006.

[27] Dionigi, M.; Mongiardo, M., "Design of via hole fed printed circular disc monopole antenna for UWB systems," in Antennas and Propagation in Wireless Communications (APWC), 2011 IEEE-APS Topical Conference on , vol., no., pp.1033-1036, 12-16 Sept. 2011.

[28] A. Singh, J. R. van Dorp, T. A. Mazzucchi, "A novel asymmetric distribution with power tails," Comm. In Statistics, Theory and Methods," 36(2), 235-252, 2007.

[29] R. Cheng, "Using Pearson type IV and other Cinderella distributions in simulations, "In Proc. Of the Winter Simulation Conference. Winter Simulation Conference, 2011.

[30] M. Z. Win, R. A. Scholtz, "Impulse radio: How it works." IEEE Communications Letters, 2(2), 36-38, 1998.

[31] Liuqing Yang; Giannakis, G.B., "Ultra-wideband communications: an idea whose time has come," in Signal Processing Magazine, IEEE , vol.21, no.6, pp.26-54, Nov. 2004

[32] H. Zhang, X. Cui, T. Aaron Gulliver, "Threshold Selection for TOA Estimation based on Skewness and Slope in Ultrawideband Sensor Networks," Journal of Networks, vol. 7, no. 7, pp. 1038-1045, July 2012.

[33] Y. Nagahara, "The PDF and CF of Pearson type IV distributions and the ML estimation of the parameters," Statistics \& Probability Letters, 43, (1999), page 251.

[34] J. Heinrich, "A Guide to the Pearson Type IV Distribution," Collider Detector at Fermilab internal note 6820, 2004, avalaible on-line: http://www-cdf.fnal.gov/publications/cdf6820 pearson4.pdf.

[35] D. Dardari, A. Conti, U. Ferner, A. Giorgetti, M. Z. Win, "Ranging with ultrawide bandwidth signals in multipath environments," Proceedings of the IEEE, 97(2), 404-426, 2009.

[36] A. De Angelis, J. O. Nilsson, I. Skog, P. Händel, P. Carbone, “ Indoor Positioning by Ultrawide Band Radio Aided Inertial Navigation”, Metr. Meas. Syst., vol. XVII, no. 3, pp. 447-460, 2010.

[37] B. Ristic, S. Arulampalam, N. Gordon, "Beyond the Kalman Filter Particle Filters for Tracking Applications", Artech House 2004;

[38] A. Conti, D. Dardari, M. Guerra, L. Mucchi, M.Z. Win, M. Z. "Experimental characterization of diversity navigation," Systems Journal, IEEE, 8(1), 115-124, 2014.

[39] D.S. Moore, and McCabe, G.P. (2003), Introduction to the Practice of Statistics, 4th ed., New York: Freeman.

[40] Thrun, W. Burgard and D. Fox, "Probabilistic Robotics", The MIT Press, 2006.

[41] B. Denis; Ouvry, L.; Uguen, B.; Tchoffo-Talom, F.; "Advanced Bayesian filtering techniques for UWB tracking systems in indoor environments " Ultra-Wideband, 2005. ICU 2005. 2005 IEEE International Conference .

[42] R. Altendorfer, S. Matzka, "A New Confidence Estimator for Vehicle Tracking Based on a Generalization of Bayes Filtering", IEEE Intelligent Transportation Systems Magazine, vol. 2 Winter 2012, pp. 30-41.

[43] M. Sanjeev Arulampalam, Simon Maskell, Neil Gordon, and Tim Clapp; "A Tutorial on Particle Filters for Online Nonlinear/NonGaussian Bayesian Tracking", IEEE Transactions on Signal Processing, vol. 50, no. 2, February 2002. 
[44] Y. Shen, M. Z. Win. "Fundamental limits of wideband localizationPart I: A general framework," Information Theory, IEEE Transactions on, 56(10), 4956-4980, 2010.

Guido De Angelis (M'07-SM'11) was born in Perugia, Italy, in 1966. He received the Laurea degree in Electronic Engineering from University of Perugia in 1993. This author became a Member (M) of IEEE in 2007, and a Senior Member (SM) in 2011. He has also received a Ph. D. in Information Engineering (Telecommunications) in 2011 from the University of Perugia, with a thesis on the study and integration of ground-based and satellitebased positioning systems. His research interests outdoor and indoor navigation system, sensor fusion, statistical signal processing, detection and estimation theory. $\mathrm{He}$ is author or co-author of papers, appearing in International Journals or Conference Proceedings. He has been serving as a Reviewer for several technical journals and international conferences. Guido De Angelis also works at Regione Umbria (Regional Government of Umbria, Italy), Office for Innovation Promotion and Enterprise Innovation Services, where he is in charge of the "ICT for SMEs" technical activities.

Antonio Moschitta (M’01) was born in 1972 in Foligno, Italy. In 1998, he obtained his Laurea degree in Electronic Engineering at the University of Perugia, Italy. In March 2002, he received his Ph.D. in Electronic Engineering, his dissertation being focused on the effects of quantization noise upon the performances of Digital Communication Systems. He is currently an assistant professor at the Department Engineering of the University of Perugia. His research interests include Power Quality, Modern Digital Communication Systems, A/D and D/A conversion, Time to Digital Conversion and Indoor Positioning. He is author or co-author or more than 60 papers, appearing in International Journals or Conference Proceedings. He is IEEE Member since 2001. He has been General coChair of the 2012 IEEE Workshop on Environmental, Energy, and Structural Monitoring Systems EESMS 2012. He is currently Chair of the IEEE Systems Council Italian Chapter.

Paolo Carbone received the Laurea and the Doctorate degrees from the University of Padova, Italy in 1990 and 1994, respectively. From 1994 to 1997 he was a researcher with the Third University of Rome. Since 2002 he is a Full Professor with the University of Perugia, where he teaches courses in Instrumentation and Measurement and in Probability Theory. Prof. Carbone has been involved in various research projects sponsored by private and public funds. He was national coordinator of a PRIN project on indoor positioning. He was chairman of the IMEKO IWADC Workshop on ADC Modeling and Testing (2003 and 2011 editions). Paolo Carbone is author/co-author of more than 150 papers, appeared in international journals (63) and conference proceedings (89) (public information about his research production on Google Scholar). He served as an Associate Editor of IEEE TRANSACTIONS ON CIRCUITS AND SYSTEMS-PART II from 2000 to 2002 and as Associate Editor of IEEE TRANSACTIONS ON CIRCUITS AND SYSTEMS-PART I from 2005 to 2007. He is the Editor-in-Chief of the international journal ACTA Imeko and VPpublications with the IEEE Systems Council, of which he is Distinguished Lecturer, since 2014 . He is a member of the steering committee of the IEEE Transactions on Cloud Computing. He is a consultant for the Italian Rectors' Confederation (CRUI) on the subject of research quality assessment. 